\title{
MoniQue GEISTDOERFER-FLORENZANO Éléments maximaux des préordres partiels sur les ensembles compacts
}

\author{
Revue française d'automatique, d'informatique et de recherche \\ opérationnelle. Recherche opérationnelle, tome 12, n 3 (1978), \\ p. 277-283. \\ <http://www.numdam.org/item?id=RO_1978_12_3_277_0>
}

(C) AFCET, 1978, tous droits réservés.

L'accès aux archives de la revue « Revue française d'automatique, d'informatique et de recherche opérationnelle. Recherche opérationnelle » implique l'accord avec les conditions générales d'utilisation (http://www.numdam.org/ legal.php). Toute utilisation commerciale ou impression systématique est constitutive d'une infraction pénale. Toute copie ou impression de ce fichier doit contenir la présente mention de copyright.

\section{Numdam}

Article numérisé dans le cadre du programme

Numérisation de documents anciens mathématiques

http://www.numdam.org/ 


\title{
ÉLÉMENTS MAXIMAUX DES PRÉORDRES PARTIELS SUR LES ENSEMBLES COMPACTS $\left({ }^{*}\right)$
}

\author{
par Moniaue Geistdooerfer-FLorenzano $\left({ }^{1}\right)$
}

\begin{abstract}
Résumé. - Cette note établit la suffisance pour l'existence, dans tout sous-ensemble quasi-compact, non vide, d'un espace topologique $X$, d'éléments maximaux pour une relation binaire asymétrique donnée sur $X$, de conditions impliquées par les conditions plus classiques trouvées dans la littérature.

Cè résultat a une application possible dans la théorie des choix quand un décideur (individu, petit groupe d'individus ou collectivité de toute taille) opère sur la base d'une préférence stricte sur un espace de choix $X$, si la famille de tous les sous-ensembles compacts non vides de $X$ représente la famille des sous-ensembles de choix potentiels auxquels l'unité de décision peut être confrontée.

D'un point de vue économique, ce résultat a des conséquences dans les problèmes d'existence de l'équilibre compétitif dans une économie où les préférences des consommateurs ne sont pas complètes.
\end{abstract}

\section{INTRODUCTION}

L'extension-de la théorie de l'équilibre économique général au cas où les préférences des consommateurs ne forment pas un préordre total sur leur ensemble de consommation s'est faite selon deux voies de recherche utilisant des techniques mathématiques différentes.

D'une part, on a longtemps considéré que la transitivité de la préférence stricte constituait une hypothèse d'un « réalisme » raisonnable et que seule devait être relaxée l'hypothèse de transitivité de l'indifférence. Les préférences de chaque consommateur constituent alors un préordre partiel sur son ensemble de consommation $X$, lequel est, au moins à ce niveau du problème, un espace topologique quelconque. La recherche de conditions suffisantes d'existence d'éléments maximaux pour le préordre partiel sur tout sous-ensemble quasi compact de $X$ est une étape nécessaire dans l'établissement de théorèmes d'existence d'un équilibre compétitif, et les conditions recherchées sont des conditions de continuité du préordre, encore appelées conditions de compatibilité du préordre partiel et de la topologie séparément donnés sur $X$.

$\left(^{*}\right)$ Reçu mars 1977, révisé janvier 1978.

( $\left.{ }^{1}\right)$ C.N.R.S., C.E.P.R.E.M.A.P., Paris.

R.A.I.R.O. Recherche opérationnelle/Operations Research, vol. 12, $\mathrm{n}^{\circ}$ 3, août 1978 
Plus récemment, la transitivité de la relation de préférence stricte a été remplacée par une hypothèse de convexité pour cette relation $\left({ }^{2}\right)$, ce qui suppose évidemment que $X$ est un espace vectoriel topologique $\left(R^{n}\right.$ ou un espace vectoriel topologique quelconque, selon le degré de généralité des résultats obtenus). L'existence d'éléments maximaux sur tout sous-ensemble convexe compact de $X$, sous les mêmes hypothèses de continuité de la relation de préférence stricte, est obtenue par application de théorèmes de point fixe.

C'est dans le premier courant de recherches, plus proche de la démarche initiale de G. Debreu dans [5] - où aucune condition de convexité n'est encore introduite au moment de l'étude de la demande - que se situe cette note. Dans la section II, nous montrerons que les conditions de continuité du préordre (partiel) les plus souvent retenues dans la littérature économique ne sont ni les seules envisageables, ni nécessairement les plus adaptées aux problèmes à traiter; des conditions plus conformes à une tradition mathématique assez ancienne $\left({ }^{3}\right)$ ont une pertinence comparable dans les problèmes d'équilibre général et garantissent les mêmes résultats. Dans la section III, nous montrerons, sous des hypothèses qui généralisent à la fois les conditions conventionnelles de continuité et celles que nous suggérons d'utiliser, l'existence d'éléments maximaux sur tout sous-ensemble quasi-compact de $X$.

\section{COMPATIBIITTÉ D'UN PRÉ ORDRE PARTIEL ET D'UNE TOPOLOGIE SUR UN ENSEMBLE $X$}

Avant d'aborder le problème, introduisons quelques notations devenues standard.

Si un ensemble $X$ est muni d'un préordre $R=(P, I)$, de partie asymétrique $P$ et de partie symétrique $I$, on note

$$
\begin{array}{ll}
P(\mathrm{x})=\{z \in X / z P x\}, & P^{-1}(x)=\{z \in X / x P z\}, \\
R(x)=\{z \in X / z R x\}, & R^{-1}(x)=\{z \in X / x R z\} .
\end{array}
$$

Un élément maximal d'un sous-ensemble $A$ de $X$ est un élément $x \in A$ tel que $P(x) \cap A=\emptyset$.

Lorsque $X$ est totalement préordonné, il est habituel d'associer au préordre la topologie $\tau^{+}$dite topologie supérieure $d u$ préordre engendrée par la famille $\mathscr{B}=\left\{P^{-1}(x) / x \in X\right\} \cdot \tau^{+}$est aussi, évidemment, la topologie la moins fine pour laquelle les ensembles $R(x)$ sont fermés. La condition $\tau \supset \tau^{+}$est une condition de continuité du préordre ou de compatibilité du préordre total $R$ et d'une topologie $\tau$ séparément donnés sur $X$.

$\left({ }^{2}\right)$ On trouvera des éléments de bibliographie dans [2].

$\left({ }^{3}\right)$ Voir les titres $[4,6,9,12$ et 13$]$ de notre bibliographie. 
Lorsque le préordre est partiel, la définition d'une topologie associée à ce préordre et qui généralise la topologie $\tau^{+}$qui vient d'être définie n'est pas univoque. Nous retiendrons deux topologies définies l'une à partir des ensembles $P^{-1}(x)$, l'autre à partir des ensembles $R(x)$. Ainsi nous désignerons comme précédemment par :

$\tau^{+}$, la topologie engendrée par la famille $\mathscr{B}=\left\{P^{-1}(x) / x \in X\right\} ;$ mais aussi par :

$\tau^{\prime+}$, la topologie engendrée par la famille $\mathscr{C}=\left\{\complement_{X} R(x) / x \in X\right\} ;$

$\tau^{+}$est la topologie la moins fine pour laquelle les ensembles $P^{-1}(x)$ sont ouverts, tandis que $\tau^{\prime+}$ est la topologie la moins fine pour laquelle les ensembles $R(x)$ sont fermés. Les conditions $\tau \supset \tau^{+}$et $\tau \supset \tau^{\prime+}$ sont des conditions de compatibilité d'un préordre et d'une topologie séparément données sur $X$ qui généralisent la condition correspondante imposée lorsque le préordre est total.

Ces deux types de conditions, qui coïncident lorsque le préordre est total, n'ont évidemment pas le même champ d'application. Par exemple, si $R^{n}$ est muni de la topologie usuelle et de l'ordre partiel : $x \leqq y \Leftrightarrow x_{i} \leqq y_{i}, \forall i=1, \ldots, n$, la topologie $\tau$ de $R^{n}$ contient $\tau^{\prime+}$ sans contenir $\tau^{+}$; inversement, si $R^{n}$ est muni de la topologie usuelle et de l'ordre partiel $(\ll,=)$ dont la relation stricte $\ll$ est définie par : $x \ll y \Leftrightarrow x_{i}<y_{i}, \forall i=1, \ldots, n$, la topologie $\tau$ de $R^{n}$ contient $\tau^{+}$sans contenir $\tau^{\prime+}$. De même, si $\lesssim_{i}, i=1, \ldots, m$ sont $m$ préordres totaux sur un même espace topologique $X$ dont la topologie $\tau$ contient les topologies $\tau_{i}^{+}$pour $i=1, \ldots, m$, le préordre (partiel) $\lesssim$ sur $X$ obtenu par la règle d'unanimité

$$
x \lesssim y \Leftrightarrow x \S_{i} y, \quad \forall i=1, \ldots, m,
$$

vérifie la condition $\tau \supset \tau^{\prime+}$, tandis que le préordre (partiel) sur $X$ obtenu par la règle d'unanimité

$$
\begin{aligned}
& x<y \Leftrightarrow x<_{i} y, \quad \forall i=1, \ldots, m, \\
& x \sim y \Leftrightarrow x \sim_{i} y, \quad \forall i=1, \ldots, m,
\end{aligned}
$$

vérifie la condition $\tau \supset \tau^{+}$.

L'intérêt de résultats fondés sur la vérification de conditions de l'un ou l'autre type dépend donc des problèmes auxquels on veut les appliquer, dans la mesure où ces problèmes déterminent le choix du préordre et de la topologie sur $X$ les plus appropriés. La littérature économique a jusqu'ici utilisé le plus souvent des conditions du premier type; nous pensons, au contraire, que l'on doit s'efforcer d'établir des résultats semblables pour les deux types de conditions. 


\section{EXISTENCE D'ÉLÉMENTS MAXIMAUX SUR TOUT SOUS-ENSEMBLE QUASI COMPACT DE $X$}

C'est sous des conditions de continuité du préordre plus faibles $\left(^{4}\right)$ que chacune des conditions $\tau \supset \tau^{+}$et $\tau \supset \tau^{\prime+}$ que nous démontrerons l'existence d'éléments maximaux sur tout sous-ensemble quasi compact $A$ de $X$. Si $A$ étant un sous-ensemble de $X, \vec{A}$ désigne l'adhérence de $A$, ces conditions s'écrivent :

$$
\begin{aligned}
& 1^{\circ} \forall x \in X, P^{-1}(x) \cap \overline{P(x)}=\varnothing ; \\
& 2^{\text {o }} \forall x \in X, u \in \overline{P(x)} \text { et } v \in P(x) \Rightarrow v \in \overline{P(x)} .
\end{aligned}
$$

L'intérêt de ces conditions est de ne porter que sur la partie asymétrique de la relation de préordre. Elles peuvent donc être utilisées dès que l'on connaît cette partie asymétrique, c'est-à-dire dès que l'on connaît une relation irréflexive et transitive sur $X$, quelle que soit la relation d'équivalence compatible avec cette relation et qui, jointe à elle, définit le préordre. Ces conditions n'impliquent d'ailleurs pas la transitivité de la relation stricte concernée et elles conservent leur sens et, comme nous allons le voir, leur efficacité pour une relation asymétrique. Elles impliquent cependant l'acyclicité de $\mathrm{P}$ :

$$
x^{1} P x^{2} P x^{3} P \ldots P x^{n} P x^{1} \Rightarrow x^{2} \in P^{-1}\left(x^{1}\right) \cap \overline{P\left(x^{-1}\right)},
$$

comme c'est prévisible pour des relations devant assurer l'existence d'éléments maximaux sur tout sous-ensemble quasi-compact de $X$ (et, en particulier, sur tout sous-ensemble fini de $X$ ). On peut voir également, directement ou indirectement via l'existence d'éléments maximaux pour tout sous-ensemble quasi-compact de $X$, que les conditions $1^{\circ}$ et $2^{\circ}$ impliquent des formes plus fortes d'acyclicité de $P$ comme celles définies par C. R. Birchenhall dans [3].

Le fait que ces conditions, appliquées à un préordre sur $X$, sont plus faibles que chacune des conditions $\tau \supset \tau^{+}$et $\tau \supset \tau^{\prime+}$ résulte des deux lemmes suivants :

LEMME 1 (J. Y. Jaffray) : Étant donné un préordre et une topologie sur un ensemble $X$, la condition $\tau \supset \tau^{+}$implique les conditions $1^{\circ}$ et $2^{\circ}$ ci-dessus.

En effet, si $y \in P^{-1}(x), P^{-1}(x)$ est un voisinage de $y$ dont l'intersection avec $P(x)$ est vide; $y$ ne peut donc appartenir à $\overline{P(x)}$. Si d'autre part $u \in \overline{P(x)}$ et si $v P u, P^{-1}(v)$ est un voisinage de $u$ qui rencontre $P(x)$; on en déduit $v P x$ et donc $v \in P(x) \subset \overline{P(x)}$.

$\left({ }^{4}\right)$ Sous ces mêmes conditions, si $X$ est un ensemble (partiellement) préordonné par $R=(P, I)$, sans trou $(y P x \Rightarrow \exists z \in X, y P z P x)$ et contenant un sous-ensemble $\mathrm{A}$, fini ou dénombrable dense pour le préordre $(y P x \Rightarrow \exists z \in X, y R z R x)$, J. Y. Jaffray dans [7] démontre l'existence d'une fonction réelle $f: X \rightarrow R$, semi-continue supérieurement et vérifiant $y P x \Rightarrow f(x)<f(y)$. L'existence, sous ces hypothèses, d'un élément maximal pour le préordre sur tout sous-ensemble quasi-compact de $X$ découle alors de l'existence sur ces mêmes ensembles d'un maximum pour $f$.

Si $X$ est sans trou et contient un sous-ensemble fini ou dénombrable dense dans $X$ pour le préordre et si $\tau \supset \tau^{+}$, B. Peleg dans [10] démontre également l'existence d'une fonction réelle $f: X \rightarrow R$ semicontinue supérieurement et vérifiant $y P x \Rightarrow f(x)<f(y)$. Ceci entraîne également l'existence d'un élément maximal dans tout sous-ensemble quasi-compact de $X$. 
Lemme $2:$ Étant donné un préordre et une topologie sur un ensemble $X$, la condition $\tau \supset \tau^{\prime+}$ implique les conditions $1^{\circ}$ et $2^{\circ}$ ci-dessus.

En effet si $y \in P^{-1}(x), \complement_{X} R(x)$ est un voisinage de $y$ dont l'intersection avec $P(x)$ est vide; $y$ ne peut donc appartenir à $\overline{P(x)}$. Si d'autre part $u \in \overline{P(x)}$, on déduit de $\overline{P(x)} \subset R(x): u \in R(x)$. Si alors $v \in P(u), v \in P(x) \subset \overline{P(x)}$.

Par ailleurs, un préordre sur $X$ peut ne vérifier aucune des conditions $\tau \supset \tau^{+}$ ou $\tau \supset \tau^{\prime+}$, alors que sa partie asymétrique vérifie les conditions $1^{\circ}$ et $2^{\circ}$. On peut, par exemple, vérifier immédiatement que l'égalité est la seule relation d'équivalence compatible avec la relation irréflexive et transitive $P$ définie sur $R^{n}$ par $x P y \Leftrightarrow r_{1}>y_{1}$ et $x_{i} \geqq y_{i}, \forall i=2, \ldots, n$. Le préordre $(P,=)$ ne vérifie aucune des conditions $\tau \supset \tau^{+}$et $\tau \supset \tau^{\prime+}$ bien que $P$ vérifie $1^{\circ}$ et $2^{\circ}$.

On notera enfin que si $R=(P, I)$ est un préordre total vérifiant $1^{\circ}$, la condition $2^{\circ}$ est automatiquement vérifiée.

Pour démontrer l'existence d'éléments maximaux pour $P$ sur tout sousensemble quasi-compact de $X$, nous utiliserons classiquement la propriété d'intersection finie qui caractérise la quasi-compacité d'un sous-ensemble $A$ de $X$ :

(P) Toute famille de sous-ensembles fermés de $A$ dont l'intersection est vide contient une sous-famille finie dont l'intersection est vide

et un théorème dit, lemme de Kuratowski, dont l'énoncé est équivalent à l'axiome du choix.

LEMME DE Kuratowski : Tout sous-ensemble totalement ordonné d'un ensemble ( partiellement) ordonné B est contenu dans un sous-ensemble totalement ordonné, maximal, pour l'inclusion des ensembles, parmi les sous-ensembles totalement ordonnés de $B$.

Le résultat auquel nous aboutissons est le suivant :

Proposition 1 : Dans un espace topologique $X$ muni d'une relation $P$ vérifiant les conditions :

$1^{\circ} \forall x \in X, P^{-1}(x) \cap \overline{P(x)}=\varnothing ;$

$2^{\circ} \forall x \in X, u \in \overline{P(x)}$ et $v \in P(u)$ impliquent $v \in \overline{P(x)}$;

tout sous-ensemble $A$ quasi-compact de $X$ contient un élément maximal pour $P$.

Soient, en effet, $A$ un sous-ensemble quasi-compact de $X$ n'admettant pas d'élément maximal et $\mathscr{F}$ la famille des ensembles fermés dans $A:(\overline{P(x)} \cap A)$ pour $x \in A$. L'inclusion dans $: \mathscr{P}(: 4)$ (ensemble des parties de $A$ ) induit sur $\mathscr{F}$ un ordre (partiel); soit alors, conformément au lemme de Kuratowski, $\mathscr{M}$ une sousfamille de $\mathscr{F}$, totalement ordonnée. maximale. Toute sous-famille finie de $/ /$ a, 
puisque $\mathscr{M}$ est totalement ordonnée, une intersection non vide. Puisque $A$ est quasi-compact, on déduit alors de $(P)$ l'existence d'un élément $x^{0}$ dans $\bigcap_{P(x) \cap A \in \mathscr{M}}(\overline{P(x)} \cap A)$.

Si alors $y \in P\left(x^{0}\right) \cap A$, il résulte de $1^{\circ}$ que $x^{0}$ n'appartient pas à $\overline{P(y)} \cap A$ et donc que $\overline{P(y)} \cap A$ n'appartient pas à $\mathscr{M}$. D'autre part, puisque $x^{0}$ appartient à tout $\overline{(P(x)} \cap A)$ de $\mathscr{M}$, il résulte de $2^{\circ}$ que pour tout $(\overline{P(x)} \cap A)$ de $\mathscr{M}$, $P(y) \subset \overline{P(x)}$ et donc que $\overline{P(y)} \cap A \subset \overline{P(x)} \cap A$.

$\mathscr{M} \cup\{\overline{P(y)} \cap A\}$ est alors une sous-famille totalement ordonnée de $\mathscr{F}$, contenant strictement $\mathscr{M}$, contrairement à l'hypothèse selon laquelle $\mathscr{M}$ est maximale. On aboutit ainsi à une contradiction, ce qui montre que tout sousensemble quasi-compact de $X$ admet un élément maximal.

Corollaire 1 (D. Schmeidler) $\left({ }^{5}\right)$ : Dans un espace topologique $X$ muni d'un préordre (partiel) vérifiant la condition $\tau \supset \tau^{+}$, tout sous-ensemble quasi-compact de $X$ admet un élément maximal.

Corollaire 2 (A. D. Wallace) $\left({ }^{6}\right)$ : Dans un espace topologique $X$ muni d'un préordre (partiel) vérifiant la condition $\tau \supset \tau^{\prime+}$, tout sous-ensemble quasi-compact de $X$ admet un élément maximal.

On remarquera pour terminer que le résultat de la proposition 1 et de ses corollaires reste vrai pour toute relation (acyclique) $Q$ prolongée par une relation $\mathrm{P}$ vérifiant les hypothèses de la proposition 1 ou de chacun des corollaires. En vertu des lemmes 1 et $2, Q$ vérifie alors la première condition de la proposition 1.

Enfin, un raisonnement symétrique de celui qui a été fait ici montrerait l'existence d'éléments minimaux dans tout sous-ensemble quasi-compact de $X$ pour une relation $P$ vérifiant des conditions symétriques des conditions de la proposition 1 ou de ses corollaires.

\section{BIBLIOGRAPHIE}

1. T. Bergstrom, Maximal Elements of Acyclic Relation on Compact Sets, J. Economic Theory, $\mathrm{n}^{\circ} 10,1975$, p. 403-404.

2. T. Bergstrom, The Existence of Maximal Elements and Equilibria in the Absence of Transitivity, University of Michigan, 1975.

$\left({ }^{5}\right)$ D. Schmeidler donne dans [11] une démonstration directe de ce résultat simplifiée par l'emploi de la propriété de recouvrement fini des ensembles compacts. T. Bergstrom reprend le résultat dans [1].

$\left({ }^{6}\right)$ L. E. Ward dans [13] attribue ce résultat à A. D. Wallace [12]. Il indique également que le résultat est signalé, sous une forme différente, par G. Birkhoff dans la deuxième édition de [4]. (1948).

R.A.I.R.O. Recherche opérationnelle/Operations Research 
3. C. R. Birchenhall, Conditions for the Existence of Maximal Elements in Compact Sets, J. Economic Theory, $\mathrm{n}^{\circ} 16,1977$, p. 111-115.

4. G. Birkhoff, Lattice Theory, American Mathematical Society, $3^{\mathrm{e}}$ édition, Providence, 1967, $1^{\text {re }}$ édition, 1940.

5. G. Debreu, Théorie de la Valeur. Analyse axiomatique de l'équilibre économique, Dunod, Paris, 1966, $1^{\text {re }}$ édition en anglais, New York, 1959.

6. S. Eilenberg, Ordered Topological Spaces, Amer. J. Math., $\mathrm{n}^{\circ} 63,1941$, p. 39-45.

7. J. Y. JAFFrAY, Semicontinuous Extension of a Partial Order, J. Math. Economics, $\mathrm{n}^{\circ} 2$, 1975, p. 395-406.

8. J. Kelley, General Topology, Van Nostrand, Princeton, 1964.

9. L. NAChBIn, Topology and Order, Princeton, 1965, $1^{\text {re }}$ édition en portugais, Chicago, 1950.

10. B. Peleg, Utility functions of Partially Ordered Topological Spaces, Econometrica, vol. $38, \mathrm{n}^{\circ} 1$, janvier 1970 , p. 93-96.

11. D. SchmeIdLer, Competitive Equilibria in Markets with a Continuum of Traders and Incomplete Preferences, Econometrica, vol. 37, $\mathrm{n}^{\circ} 4$, octobre 1969, p. 578-585.

12. A. D. Wallace, A Fixed Point Theorem, Bull. Amer. Math. Soc., $\mathrm{n}^{\circ} 51,1945$, p. $413-$ 416.

13. L. E. WARD, Partially Ordered Topological Spaces, Proc. Amer. Math. Soc., $\mathrm{n}^{\circ} 5$, 1954, p. 144-161. 